\title{
Capacidade Aeróbia de Ratos Alimentados Com Dieta Rica em Frutose
}

\section{Aerobic Capacity of Rats Fed With Fructose Rich Diet}

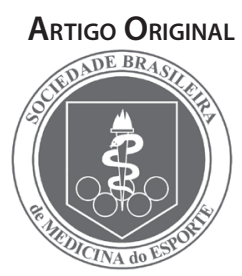

\author{
Rodrigo Ferreira de Moura \\ Lucieli Teresa Cambri \\ Antonio Carlos de Quadros Junior \\ Carla Manuela Crispim Nascimento \\ Luciana Mendonça Arantes \\ Emerson Sebastião \\ Kátia Tanaka \\ Camila Bosquiero Papini \\ Merlyn Mércia Oliani \\ Clécia Soares de Alencar Mota \\ Carla Ribeiro \\ Maria Alice Rostom Melo.
}

Universidade Estadual Paulista - UNESP - Rio Claro - Instituto de Biociências - Departamento de Educação Física.

Programa de Pós-Graduação em

Ciências da Motricidade

\section{Endereço para correspondência:}

Maria Alice Rostom Mello

UNESP - Instituto de Biociências

- Departamento de Educação Física Avenida 24 A, 1515

Bela Vista - Rio Claro/SP

CEP 13506-900

Telefone: (19) 3526-4320

Fax: (19) 3534-6436

E-mail: mellomar@rc.unesp.br

Submetido em 27/09/2007

Versão final recebida em 14/03/2008 Aceito em 04/04/2008

\begin{abstract}
RESUMO
Introdução: Evidências apontam que a ingestão exacerbada de frutose pode desencadear distúrbios característicos da síndrome metabólica. Objetivos: Analisar os efeitos da ingestão de dieta rica em frutose sobre aspectos metabólicos de ratos da linhagem Wistar. Adicionalmente, verificar a capacidade aeróbia através da identificação da máxima fase estável de lactato (MFEL). Métodos: Dezesseis ratos foram separados em dois grupos de oito animais: a) controle, alimentados com dieta balanceada, e b) frutose, alimentados com dieta rica em frutose. Foram analisadas a tolerância à glicose (área sob a curva de glicose durante teste de tolerância à glicose), sensibilidade à insulina (taxa de remoção da glicose sérica após sobrecarga exógena de insulina), perfil lipídico sérico e concentração de lactato sanguíneo [lac]s durante exercício na intensidade da MFEL. Resultados: Teste $t$ não pareado $(p<0,05)$ revelou diferença para a tolerância à glicose e triglicérides, porém não houve diferença na sensibilidade à insulina e na [lac]s. Anova one way com post hoc de Newman-Keuls $(p<0,05)$ revelou diferença para a cinética da glicose durante o teste de tolerância, mas não para a cinética do lactato durante exercício na MSSL. Conclusão: As alterações fisiológicas provocadas pela dieta rica em frutose e inerentes à síndrome metabólica não prejudicam a capacidade aeróbia de ratos.
\end{abstract}

Palavras-chave: máxima fase estável de lactato, síndrome metabólica, exercício.

\begin{abstract}
Introduction: Evidence points that exacerbated ingestion of fructose may trigger disturbs characteristic of the metabolic syndrome. Objectives: To analyze the effects of a fructose rich diet on metabolic aspects of Wistar lineage rats. Additionally, to verify the aerobic capacity, through the identification of the maximal lactate steady state (MSSL). Procedures: Sixteen rats were separated in two groups of eight animals: a) Control, fed a balanced diet, and b) fructose, fed a fructose-rich diet. The glucose tolerance, (area under serum glucose during a glucose tolerance test), insulin sensibility (glucose disappearance rate after exogenous insulin administration), serum lipid profile and blood lactate concentration [lac]b during exercise at MSSL intensity, have been analyzed. Results: Non-paired $t$ test $(p<0.05)$ revealed difference between groups in the area under the curve of glucose and serum triglycerides, no difference in insulin sensibility or in [lac]b was detected, though. One-way ANOVA with Newman Keuls post hoc revealed difference in the glucose kinetics during tolerance test, but not in the lactate kinetics during the MSSL. Conclusion: The physiological alterations promoted by fructose-rich diet and intrinsic to the metabolic syndrome do not harm the aerobic capacity of rats.
\end{abstract}

Keywords: maximum steady state lactate, metabolic syndrome, exercise.

\section{INTRODUÇÃO}

O excesso de ingestão de frutose nos hábitos alimentares da sociedade contemporânea ${ }^{(1)}$ tem despertado interesse nos pesquisadores da área da saúde, pois foi reportado que, em ratos, dieta rica em frutose promove anormalidades metabólicas, como a hiperinsulinemia, resistência à insulina e dislipidemia ${ }^{(2,3)}$.

A associação de dislipidemia, diabetes mellitus tipo 2 ou intolerância à glicose, hipertensão arterial e obesidade caracterizam a síndrome metabólica ${ }^{(4)}$. Esses múltiplos fatores de risco aceleram a incidência das doenças coronarianas ${ }^{(4-6)}$. A hipótese fisiopatológica que mais amplamente unifica os diversos itens da síndrome metabólica é interação entre obesidade e resistência à insulina.

Uma vez que, os modelos animais mimetizam diversas respostas fisiológicas humanas, ratos alimentados com frutose têm sido utilizados como modelo experimental da síndrome metabólica humana ${ }^{(7,8)}$.

A maioria dos trabalhos ${ }^{(7,9-16)}$ analisa a influência da elevada ingestão de frutose somente em parâmetros de repouso. Porém, o exercício físico é um componente importante na prevenção e no tratamento da síndrome; foi demonstrado que o nível de capacidade aeróbia apresenta relação inversa com o risco de desenvolver essa doença ${ }^{(17)}$. Adicionalmente, a atividade física pode atenuar a gravidade do diabetes tipo 2 e melhorar a tolerância à glicose ${ }^{(18)}$.

Porém, diante das diversas alterações morfofisiológicas, provocadas por dieta rica em frutose, torna-se necessário investigar se a capacidade aeróbia também pode ser prejudicada, haja vista que é componente fundamental na prescrição de exercícios. 
Desse modo, o objetivo do presente estudo foi analisar as características metabólicas de ratos submetidos ao tratamento com dieta rica em frutose. Adicionalmente, verificar a influência dos possíveis distúrbios metabólicos, provocados pela dieta, na capacidade aeróbia inferida pela máxima fase estável de lactato sanguíneo (MFEL).

\section{MÉTODOS}

\section{Animais e seu tratamento}

Todos os experimentos com os animais foram realizados de acordo com as resoluções brasileiras específicas sob a Bioética em Experimentos com Animais (Lei n 6.638, de 8 de maio de 1979 e Decreto ${ }^{\circ}$ 24.645, de 10 julho de 1934).

O estudo foi realizado com 16 ratos da linhagem Wistar, recémdesmamados. Os animais foram mantidos em gaiolas coletivas de polietileno, à temperatura de $25 \pm 1^{\circ} \mathrm{C}$ e em ciclo claro/escuro de 12/12 horas, com livre acesso à água e ao alimento.

\section{Tratamento dietético}

A partir do desmame (21 dias) até a idade adulta (90 dias), o alimento consistiu de dietas semipurificadas balanceadas - AIN-93G ${ }^{(19)}$, ou compostas por $60 \%$ de frutose, conforme composição descrita na tabela 1.

Tabela 1. Composição das dietas.

\begin{tabular}{l|c|c}
\multicolumn{1}{c|}{ Componentes (g/kg) } & Balanceada & Rica em frutose (60\%) \\
\hline Caseína & 202 & 202 \\
\hline Amido & 397 & - \\
\hline Dextrina & 130,5 & - \\
\hline Sacarose & 100 & 27,5 \\
\hline Frutose & - & 3 \\
\hline L-cistina & 3 & 70 \\
\hline Óleo de soja & 70 & 2,5 \\
\hline Mistura de sais (AIN-93GMX) & 35 & 35 \\
\hline Mistura de vitaminas (AIN-93GVX) & 10 & 10 \\
\hline Floridrato de colina & 50,5 & 30 \\
\hline
\end{tabular}

\section{Grupos experimentais}

Após o desmame até a idade adulta, os animais foram separados em dois grupos de tratamento compostos por oito ratos cada:

- Controle (GC): alimentados com dieta balanceada;

- Frutose (GF): alimentados com a dieta rica em frutose.

\section{Avaliações gerais:}

Todos os animais tiveram o peso corporal, ingestão alimentar e hídrica registrados uma vez por semana. No dia do sacrifício, o índice de Lee ${ }^{(20)}$, um indicativo de sobrepeso, foi calculado para os dois grupos pela equação:

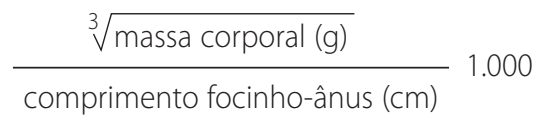

\section{Teste de tolerância à glicose - GTTo}

O GTTo foi realizado com os animais aos 90 dias de idade, após 15 horas de jejum. Os ratos foram moderadamente anestesiados com éter etílico e uma primeira coleta de sangue foi feita através de corte na extremidade da cauda do animal (tempo 0). Em seguida, uma solução de glicose a 20\% (2g/kg de massa corporal) foi administrada aos ratos por sonda gástrica de polietileno. Amostras de sangue foram coletadas após 30, 60 e 120 minutos com capilares heparinizados e calibrados para $25 \mu \mathrm{L}$, visando a determinação das concentrações de glicose.

As concentrações de glicose sanguínea foram determinadas pelo método enzimático colorimétrico da glicose oxidase-peroxidase (21), com kit Wiener Lab. Após 15 minutos de incubação em banho a $37{ }^{\circ} \mathrm{C}$, as absorbâncias das amostras e do padrão foram lidas em espectrofotômetro a 505nm. A área sob a curva da glicose(22) foi calculada utilizando-se o software Origin 3.5.

\section{Teste de tolerância à insulina - ITT}

O ITT foi realizado com os animais 48 horas após o GTTO. Os ratos foram moderadamente anestesiados com éter etílico e uma primeira coleta de sangue foi feita através de corte na extremidade da cauda do animal (tempo 0). Em seguida, foi administrada por via subcutânea uma solução de insulina cristalina Lilly U 40 na dose de $30 \mathrm{mU} / 100 \mathrm{~g}$ de massa corporal do animal. Amostras de sangue foram coletadas após 30, 60 e 120 minutos com capilares heparinizados e calibrados para $25 \mu \mathrm{L}$, visando a determinação das concentrações de glicose.

As concentrações de glicose sanguínea foram determinadas pelo método enzimático colorimétrico da glicose oxidase-peroxidase, com kit Wiener Lab. Após 15 minutos de incubação em banho a 370 C, as absorbâncias das amostras e do padrão foram lidas em espectrofotômetro a $505 \mathrm{~nm}$. A constante de redução da glicemia (K-ITT) foi calculada utilizando-se o software Origin 3.5.

\section{Teste de esforço}

Aos 90 dias de idade, todos os ratos foram submetidos a testes de esforço para a identificação do limiar de transição entre os metabolismos aeróbio e anaeróbio - MFEL. Esse limiar equivale à mais alta concentração de lactato sanguíneo [lac]s, onde sua entrada na corrente sanguínea é compensada pela remoção durante exercícios com intensidade constante ${ }^{(23)}$. A determinação da MFEL é útil para a prescrição de exercícios e avaliação do condicionamento aeróbio.

Os ratos foram submetidos a testes de nado de característica retangular após quatro dias de adaptação à água. As sobrecargas foram aplicadas em dias alternados, com intervalo mínimo de 48 horas; para isso foram utilizadas pequenas bolsas com chumbo, atadas ao tórax, que variaram entre 4 a $8 \%$ da massa corporal, com 0,5\% para a unidade mínima da variação. A duração dos testes foi de 25 minutos, com coletas de $25 \mu \mathrm{l}$ de sangue da extremidade da cauda a cada cinco minutos, para dosagem de lactato pelo método de espectrofotometria.

O critério para determinação da MFEL foi a carga de trabalho em que a diferença dos valores de lactato, entre o $25^{\circ}$ e o $10^{\circ}$ minutos de exercício, era inferior a $1 \mathrm{mM}$. A [lac]s correspondente à MFEL foi assumida como a média dos valores entre o $10^{\circ}$ e $25^{\circ}$ minutos ${ }^{(24)}$. 


\section{SACRIFÍCIO DOS ANIMAIS E OBTENÇÃO DE MATERIAL BIOLÓGICO}

\section{- Sangue}

Ao final do experimento, os animais foram sacrificados por decapitação, 48 horas após a última avaliação in vivo e após jejum de uma noite e em repouso. O sangue foi coletado e a separação do soro realizada para a dosagem de glicose, triglicérides, colesterol total, colesterol $\mathrm{LDL}$, colesterol HDL, por métodos colorimétricos ${ }^{(25)}$.

\section{- Tecido adiposo}

O tecido adiposo das regiões subcutânea posterior, mesentérica e retroperitonial foi removido para pesagem. A excisão dos diferentes depósitos de gordura foi realizada de acordo com a descrição de Cinti ${ }^{(26)}$.

\section{- Análise estatística}

Foi aplicado teste de Shapiro-Wilk para verificar a normalidade dos dados e teste $t$ não pareado para comparar entre os grupos: a média de ingestão alimentar e hídrica, a área sob a curva do GTTo, o K-ITT, a média de carga correspondente à MFEL e da [lac]s, a glicemia basal, os lipídios séricos, o peso do tecido adiposo, a massa corporal e o índice de Lee.

Para comparar o padrão da curva do GTTo e a cinética do lactato sanguíneo na carga de MFEL, de ambos os grupos, foi utilizada Anova one way para medidas repetidas e, quando necessário, análise post hoc de Newman-Keuls. O nível de significância preestabelecido em todas as análises foi de $p<0,05$.

\section{RESULTADOS}

Não foram encontradas diferenças significativas para a média de ingestão alimentar (GC: 6,43 $\pm 2,48$ vs. GF: 6,24 2,23g/100g de massa corporal) e ingestão hídrica (GC: 10,19 $\pm 3,15$ vs. GF: 8,0 $\pm 2,20 \mathrm{~g} / 100 \mathrm{~g}$ de massa corporal) diárias. Não houve diferença entre as cargas de MFEL encontradas (GC: 5,7 \pm 0,9 vs. GF: 5,6 $\pm 1,1 \%$ da massa corporal). O GF apresentou maior área sob a curva de glicose e padrão de resposta glicêmica em comparação com o GC. O perfil glicêmico durante o GTTo é apresentado na figura 1.

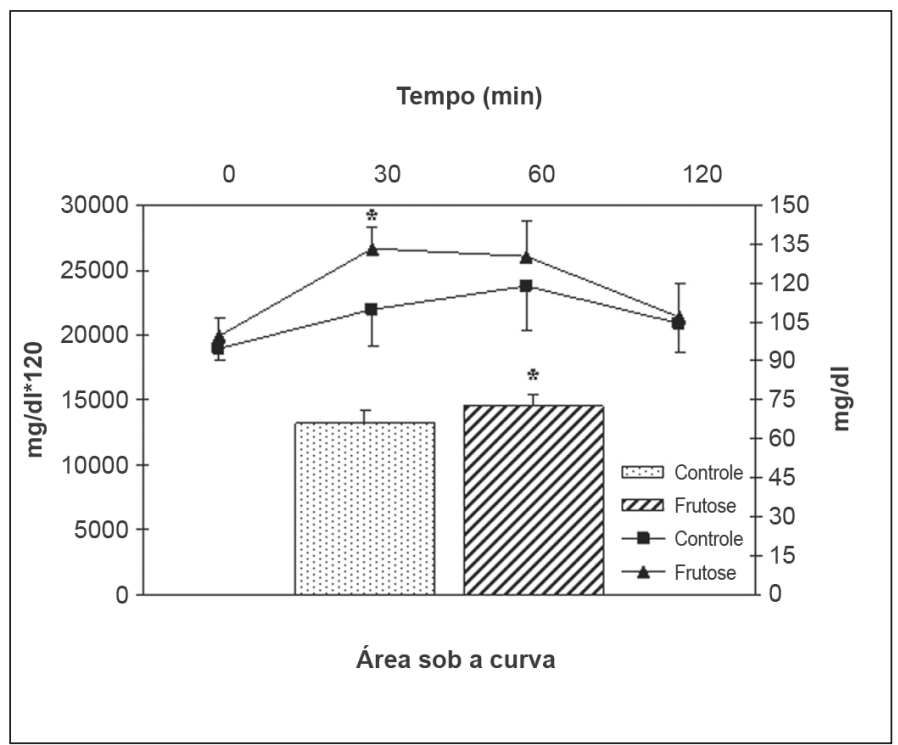

Figura 1. Glicemia e área sob a curva da glicose sérica durante teste de tolerância à glicose (média \pm desvio-padrão). * diferente do controle $(p<0,05)$.
A sensibilidade à insulina foi semelhante nos dois grupos, conforme evidenciado através do K-ITT (GC: 0,69 0,15 vs. GF: 0,72 \pm 0,35\%. $\mathrm{min}^{-1}$ ). A MFEL determinada para os animais de ambos os grupos variou de 4 a $7 \%$ do peso corporal e não houve diferença entre as concentrações médias de lactato durante exercício na intensidade da MFEL (GC: 4,97 $\pm 1,36$ vs. GF: $5,59 \pm 1,55 \mathrm{mmol} / \mathrm{l})$.

A cinética do lactato sanguíneo do GF não foi diferente do GC; a figura 2 apresenta o comportamento do lactato sanguíneo durante exercício na intensidade da MFEL.

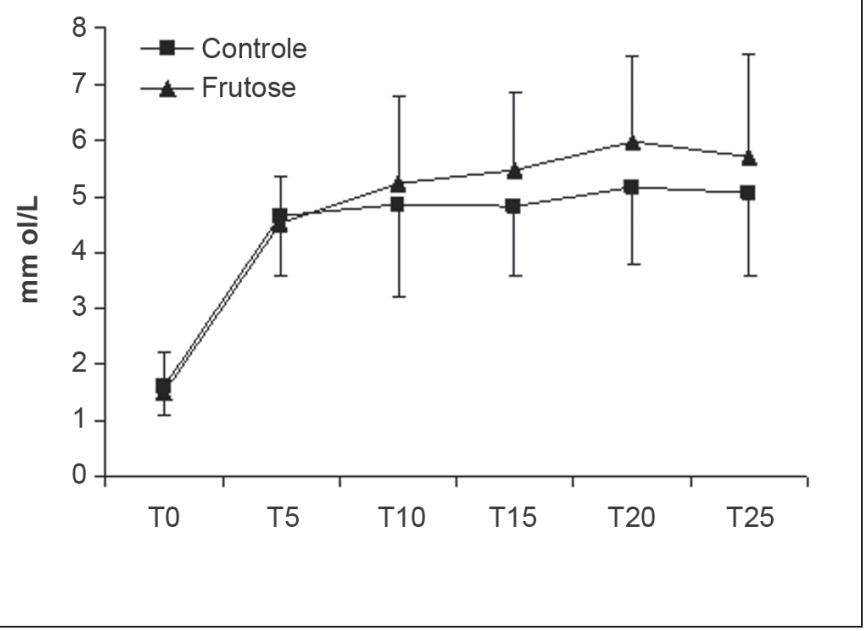

Figura 2. Cinética do lactato sanguíneo na intensidade da máxima fase estável de lactato (média \pm desvio-padrão).

Dados referentes aos lipídios séricos, peso do tecido adiposo em diferentes regiões, massa corporal e índice de Lee são apresentadas na tabela 2. A concentração de triglicérides séricos do GF foi maior do que a encontrada no GC; as demais variáveis não apresentaram diferenças significativas.

Tabela 2. Glicose e lipídios séricos, peso do tecido adiposo de diferentes regiões, massa corporal e índice de Lee dos animais ao final do experimento (90 dias de idade).

\begin{tabular}{l|c|c}
\hline & Controle & Frutose \\
\hline Glicemia basal (mg/dl) & $127,3 \pm 14,9$ & $120,6 \pm 15,9$ \\
\hline Triglicérides séricos (mg/dl) & $60,6 \pm 17,1$ & $96,2 \pm 22,3 *$ \\
\hline Colesterol total sérico (mg/dl) & $95,6 \pm 22,8$ & $116,5 \pm 19,7$ \\
\hline Colesterol HDL sérico (mg/dl) & $37,7 \pm 10,3$ & $41,8 \pm 11,6$ \\
\hline Colesterol LDL sérico (mg/dl) & $30,6 \pm 12,7$ & $32,0 \pm 16,8$ \\
\hline Gordura subcutânea posterior (g/100g animal) & $0,77 \pm 0,35$ & $0,64 \pm 0,20$ \\
\hline Gordura mesentérica (g/100g animal) & $0,76 \pm 0,23$ & $0,75 \pm 0,18$ \\
\hline Gordura retroperitonial (g/100g animal) & $0,71 \pm 0,29$ & $0,74 \pm 0,25$ \\
\hline Massa corporal (g) & $422,6 \pm 50,8$ & $426,7 \pm 45,1$ \\
\hline Indice de Lee & $298,8 \pm 7,65$ & $307,9 \pm 10,6$ \\
\hline
\end{tabular}

* Diferente do grupo controle $(p<0,05)$; valores expressos em média \pm desvio-padrão 


\section{DISCUSSÃO}

O aumento no consumo alimentar de frutose coincide com a crescente prevalência de obesidade e síndrome metabólica nas duas últimas décadas ${ }^{(27)}$. Foi demonstrado que uma dieta rica em frutose em humanos e animais de laboratório pode afetar o metabolismo de carboidratos e lipídios ${ }^{(28)}$. Essas alterações provocam distúrbios característicos da síndrome metabólica, a qual foi primeiramente descrita por Reaven ${ }^{(4)}$. A ocorrência dos componentes dessa patologia aumenta o risco de desenvolvimento de diabetes tipo 2, aterosclerose, doenças cardiovasculares e renais ${ }^{(4,5)}$.

O modelo de síndrome metabólica induzida em ratos pela ingestão de elevado teor de frutose caracteriza-se pela hipertensão, hiperinsulinemia, hipertrigliceridemia e resistência à insulina ${ }^{(2,4)}$. Foi reportado que esse modelo experimental induz a resistência à insulina com perfil metabólico muito similar ao da síndrome observada em humanos ${ }^{(2)}$.

No presente estudo, o peso corporal dos ratos não diferiu entre os grupos, o que coincide com achados anteriores $(3,10,11,25,28-30)$, assim como a ingestão de alimento ${ }^{(15,20)}$. De fato, poucas investigações relatam o incremento no peso corporal devido à dieta rica em frutose ${ }^{(13,31)}$.

A ausência de diferenças no ganho de peso corporal, assim como na quantidade de tecido adiposo nas regiões mensuradas, demonstra que a dieta apresentada não foi eficaz na indução da obesidade. Esse fato é importante, pois, de acordo com os atuais critérios para diagnóstico da síndrome metabólica, propostos pela Federação Internacional de Diabetes ${ }^{(32)}$, a obesidade central é característica indispensável. Essa limitação do modelo pode estar associada à linhagem dos ratos adotada no presente estudo, pois o ganho excessivo de peso em função de elevada ingestão de frutose foi relatada para ratos da linhagem Sprague Dawley(33).

Corroborando estudos prévios ${ }^{(10,34)}$, a elevada ingestão de frutose ocasionou intolerância à glicose, o que pode ter sido mediado pela redução na translocação dos transportadores de glicose do meio intracelular para a membrana, já que a resistência à insulina não foi alterada ${ }^{(35)}$.

Contrariamente aos nossos achados, Bezerra et al. ${ }^{(11)}$ relataram resistência à insulina ao observar a constante de decaimento da glicose após administração endovenosa daquele hormônio. Adicionalmente, Joyeux-Faure et al. ${ }^{(14)}$ aplicaram a técnica de clamp euglicêmico-hiperinsulinêmico em ratos Wistar e também constataram redução na sensibilidade à insulina. Uma possibilidade a ser investigada para esse tipo de modelo seria menor sensibilidade do ITT com administração subcutânea em comparação com a endovenosa. Contudo, uma vez que, a resistência à insulina pode ser conseqüência natural da intolerância à glicose, infere-se que a exposição continuada à dieta somada aos efeitos deletérios do envelhecimento culminaria nesse distúrbio adicional.

A elevação nas concentrações de triglicérides constatada no presente trabalho é quase unanimidade, nos mais diferentes estudos ${ }^{3,9-}$ $11,13,15,16,22,25,28,34)$; em alguns destes, tais modificações foram igualmente observadas no fígado ${ }^{(13,36)}$.

Altos níveis de triglicérides séricos são descritos como conseqüência da ingestão de frutose ${ }^{(28)}$, uma vez que esta é mais lipogênica que a glicose ${ }^{(3)}$. Dentre os mecanismos sugeridos para explicar esse fenômeno, destaca-se o aumento da lipogenêse hepática com elevada produção de VLDL ${ }^{(27)}$. Contrariamente aos nossos achados, alguns estudos evidenciaram também aumento no colesterol total(22,28). No entanto, os efeitos da frutose no metabolismo do colesterol total não estão bem claros $^{(11,29)}$
Em linhas gerais, esse padrão alimentar induz uma resposta estressante ao fígado e outros tecidos, que respondem com resistência à insulina e desregulação no metabolismo de lipídios ${ }^{(28)}$. Sánchez-Lozada et al. ${ }^{(7)}$ verificaram relação direta entre o aumento na proporção de frutose ingerida na dieta com a piora nas características da síndrome metabólica (controle < frutose 10\% < frutose 60\%).

As discrepâncias com outros estudos relacionadas à indução de obesidade ${ }^{(13,31)}$ e resistência à insulina ${ }^{(11,14)}$ também podem estar relacionadas à linhagem dos animais adotados e ao tempo de exposição à dieta. Os períodos de administração da dieta descritos na literatura variaram de duas a 13 semanas $^{(7,11-16,28,30,31,33)}$; muitos desses estudos

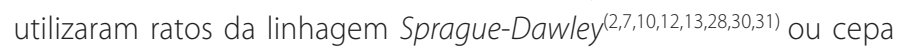
SHR - ratos espontaneamente hipertensos ${ }^{(37)}$.

A resposta do lactato sanguíneo durante o teste de esforço permitiu determinar a MFEL em ambos os grupos. Gobatto et al. ${ }^{(24)}$ relataram que em ratos eutróficos a MFEL ocorreu quando os animais exercitaram-se suportando sobrecargas equivalentes a 5-6\% do peso corporal e foram encontradas concentrações médias de lactato em 5,5mmol/L.

No presente estudo, para as cargas correspondentes à MFEL não houve diferenças entre os grupos, o que indica que os animais alimentados com frutose não tiveram sua capacidade aeróbia prejudicada. Ainda, em exercício na carga de trabalho referente à transição dos metabolismos aeróbio/anaeróbio, apresentaram respostas fisiológicas similares quanto à cinética e [lac]s final. Pode-se inferir que os mecanismos de produção e/ou remoção do lactato não foram afetados ou de alguma maneira contrabalanceados.

Na metabolização da frutose ingerida, a maior parte é extraída pelos tecidos esplânicos, nos quais pode ser convertida em glicose, lipídios ${ }^{(38)}$ ou lactato ${ }^{(39)}$. A ausência de diferenças na glicemia basal e no lactato sanguíneo aponta para a via lipogênica do metabolismo como preferencial organismo de ratos sedentários.

O nível de capacidade aeróbia de humanos adultos ${ }^{(40)}$ e crianças $^{(41)}$ apresenta relação inversa com o risco de desenvolver a síndrome metabólica ${ }^{(18)}$. Provavelmente, essa relação é o reflexo de um estilo de vida fisicamente ativo, através do qual pode desenvolver-se capacidade aeróbia mais elevada. Os achados de Hittel et al. ${ }^{(39)}$ corroboram essa hipótese, pois reportaram que a prática regular de atividade física promove alterações na atuação de algumas enzimas-chaves do sistema metabólico. Tais alterações são descritas como capazes de prevenir e atenuar os efeitos da síndrome metabólica ${ }^{(39)}$.

A constatação de que a capacidade aeróbia não é prejudicada na presença de algumas alterações fisiológicas inerentes à síndrome metabólica tem especial importância para a prescrição de atividade física para os grupos de risco. O fato de o metabolismo da frutose não alterar a MFEL indica que esse parâmetro também pode ser empregado, em estudos futuros, para determinar a intensidade e o volume adequados de exercício.

Pode-se concluir que as alterações na tolerância à glicose e nos triglicérides séricos, provocados pela dieta rica em frutose, não prejudicam a capacidade aeróbia de ratos.

\section{AGRADECIMENTOS}

Os autores agradecem a Clarice Sibuya, José Roberto Rodrigues e Eduardo Custódio pela contribuição técnica. Suporte financeiro: Fapesp processo 07/54098-0; CNPq processo 30027/2004-6.

Todos os autores declararam não haver qualquer potencial conflito de interesses referente a este artigo. 


\section{REFERÊNCIAS BIBLIOGRÁFICAS}

1. Gross LE, Li L, Ford ES, Liu S. Increased consumption of refined carbohydrates and the epidemic of type 2 diabetes in the United States: an ecologic assessment. Am J Clin Nutr 2004;79:774-9.

2. Dai S, McNeill JH. Fructose-induced hypertension in rats is concentration-and duration-dependent. J Pharmacol Toxicol Methods 1995;33:101-7.

3. Katakam PV, Ujhelyi MR, Hoenig ME, Miller AW. Endothelial dysfunction precedes hypertension in diet-induced insulin resistance. Am J Physiol 1998;275:R788-92.

4. Reaven GM. Banting lecture. Role of insulin resistance in human disease. Diabetes 1988;37:1595-307

5. Fujioka S, Matsuzawa Y, Tokunaga K, Tarui S. Contribution of intra-abdominal fat accumulation to the impairment of glucose and lipid metabolism in humam obesity. Metabolism 1987;36:54-9.

6. Kaplan NM. The deadly quartet: ipperbody obesity, glucose intolerance, hyperglycemia and hypertension. Arch Inter Med 1989;149:1514-20.

7. Sánchez-Lozada LG, Tapia E, Jiménez A, Bautista P, Cristóbal M, Nepomuceno T, et al. Fructose-induced metabolic syndrome is associated with glomerular hypertension and renal microvascular damage in rats. AJP Renal Physiology 2007;292:422-9.

8. Rayssiguier Y, Gueux E, Nowacki W, Rock E, Mazur A. High fructose consumption combined with low dietary magnesium intake may increase the incidence of the metabolic syndrome by inducing inflammation. Magnes Res 2006;19:237-43.

9. Barbosa CR, Albuquerque EMV, Faria EC, Oliveira HCF, Castilho LN. Opposite lipemic response of Wistar rats and C57BL/6 mice to dietary glucose or fructose Supplementation. Braz J Med Biol Res 2007:40:323-31

10. Sharabi Y, Oron-Herman M, Kamari Y, Avni I, Peleg E, Shabtay Z, et al. Effect of PPAR-y agonist on adiponectin levels in the metabolic syndrome: lessons from the high fructose fed rat model. Am J Hypertens 2007;20:206-10.

11. Bezerra RMN, Ueno M, Silva MS, Tavares DQ, Carvalho CRO, Saad MJA, et al. A high-fructose diet induces insulin resistance but not blood pressure changes in normotensive rats. Braz J Med Biol Res 2001;34:1155-60

12. Lee $Y, K_{0} Y$, Hsu Y, Ho L. Plasma leptin response to oral glucose tolerance and fasting/re-feeding tests in rats with fructose-induced metabolic derangements. Life Sciences 2006;78:1155-62.

13. Wang $X$, Hattori Y, Satoh H, Iwata C, Banba N, Monden T, et al. Tetrahydrobiopterin prevents endothelial dysfunction and restores adiponectin levels in rats. Eur J Pharmacol 2007:555:48-53.

14. Joyeux-Faure M, Rossini E, Ribuot C, Faure P. Fructose-fed rat hearts are protected against ischemiareperfusion injury. Exp Biol Med 2006;231:456-62.

15. Oron-Herman M, Rosenthal T, Mirelman D, Miron T, Rabinkov A, Wilchek M, et al. The effects of S-allylmercaptocaptopril, the synthetic product of allicin and captopril, on cardiovascular risk factors associated with the metabolic syndrome. Atherosclerosis 2005;183:238-43.

16. Hwang, IS, Ho, H Hofaman BB, Reave GM. Fructose-induced insulin resistance and hypertension in rats. Hypertension 1987;10:512-6.

17. Brien SE, Janssen I, Katzmarzyk PT. Cardiorespiratory fitness and metabolic syndrome: US National Health and Nutrition Examination Survey 1999-2002. Appl Physiol Nutr Metab 2007;32:143-7.

18. Lakka TA, Laaksonen DE. Physical activity in prevention and treatment of the metabolic syndrome Appl Physiol Nutr Metab 2007;32:76-88.

19. Reeves PG, Nielsen FH, Fahey-Jr GC. AlN-93 Purified diets for laboratory rodents: final report of the American Institute of Nutrition ad hoc writing committee on the reformulation of the AIN-76A rodent diet. J Nut 1993;123:1939-51.

20. Bernardis LL, Petterson BD. Correlation between "Lee index" and carcass fat content in weanling and adult female rats wth hypothalamic lesions. J Endocrinology 1968:40:527-8.
21. Henry RJ, Cannon DC, Wilkeman J. Clinical Chemistry, principles and techniques. Harper and Harper Row Publishes. New York, 1974

22. Mathews JNS, Altaman DG, Campbell MJ, Royston P. Analysis of serial measurements in medical research. Br Med J 1990;27:230-5.

23. Heck H, Mader A, Hess G, Muck S, Hollman W. Justification for $4.0 \mathrm{Mmol} / \mathrm{L}$ lactate threshold. Int. J. Sports Med 1985;6:117-30

24. Gobatto CA, Mello MAR, Sibuya CY, Azevedo JRM, Santos LA, Kokubun E. Maximal lactate steady state in rats submitted to swimming exercise. Comp Biochem Physiol 2001;130:21-7.

25. Nogueira DM, Strufaldi B, Hirata MH, Abdalla DSP, Hirata RDC. Sangue-Parte I: Glicídios. In: Nogueira DM, Strufaldi B, Hirata MH, Abdalla DSP, Hirata RDC, editores. Métodos de Bioquímica Clínica, São Paulo, 1990

26. Cinti S. The adipose organ. Prostaglendins, Leukotrienes and Essential Fatty Acids 2005;73:9-15

27. Elliott SS, Keim NL, Stern JS, Teff K, Havel PJ. Fructose, weight gain, and the insulin resistance syndrome. Am J Clin Nutr 2002;76:911-22.

28. Kelley $\mathrm{GL}$, Allan G, Azhar S. High dietary fructose induces a hepatic stress response resulting in cholesterol and lipid dysregulation. Endocrinol 2004;145:548-55.

29. Girard A, Madani S, Boukortt F, Cherkaoui-Malki M, Belleville J, Prost J. Fructose-enriched diet modifies antioxidant status and lipid metabolism in spontaneously hypertensive rats. Nutrition 2006;22:758-66

30. Roglans R, Vila L, Farré M, Alegret M, Sánchez RM, Vázquez-Carrera M, et al. Impairment of hepatic stat-3 activation and reduction of PPAR activity in fructose-fed rats. Hepatology 2007;45:778-88.

31. Jürgens $H$, Haass W, Castañeda TR, Schürmann A, Koebnick C, Dombrowski F, et al. Consuming fructose-sweetened beverages increases body adiposity in mice. Obes Res 2005;13:1146-56.

32. International Diabetes Federation. The IDF consensus worldwide definition of the metabolic syndrome. http://www.idf.org/webdata/docs/IDF_Meta_def_final.pdf. 2005.

33. Kanarek RB, Orthen-Gambill N. Differential effects of sucrose, fructose and glucose on carbohydrate induced obesity in rats. J Nutr 1982;112:1546-54

34. Thorburn AW, Storlien $\mathrm{LH}_{\text {, Jenkins } A B}$, Khouri S, Kraegen EW. Fructose-induced in vivo insulin resistance and elevated plasma triglyceride levels in rats. Am J Clin Nutr 1989;49:155-63.

35. Pereira LO, Francishchi RP, Lancha Jr AH. Obesidade: hábitos nutricionais, sedentarismo e resistência à insulina. Arq Bras Endocrinol Metab 2003;47:111-27.

36. Zavaroni I, Bonora E, Pagliara M, Dall'Aglio E, Luchetti L, Buonanno G, et al. Risk factors for coronary artery disease in healthy persons with hyperinsulinemia and normal glucose tolerance. N Engl J Med 1989;320:702-6.

37. Cesaretti MLR, Kohlmann-Jr O. Modelos experimentais de resistência à insulina e obesidade: lições aprendidas. Arq Bras Endocrinol Metab 2006;50:190-7

38. Mayes PA. Intermediary metabolism of fructose. Am J Clin Nutr 1993;58:7545-55.

39. Hittel DS, Kraus WE, Tanner CJ, Houmard JA, Hoffman EP. Exercise training increases electron and substrate shuttling proteins in muscle of overweight men and women with the metabolic syndrome. J Appl Physiol 2005:98:168-79.

40. Borodulin K, Laatikainen T, Lahti-Koski M, Lakka TA, Laukkanen R, Sarna S, et al. Associations between estimated aerobic fitness and cardiovascular risk factors in adults with different levels of abdomina: obesity. Eur J Cardiovasc Prev Rehabil 2005;12:126-31.

41. Rizzo NS, Ruiz JR, Hurtig-Wennlof A, Ortega FB, Sjostrom M. Relationship of physical activity, fitness, and fatness with clustered metabolic risk in children and adolescents: the European youth heart study. J Pediatr 2007:150:388-94. 